Microscopy

Coming Events

2019

National Society for Histology, 45th

Annual Symposium

September 20-25, 2019

New Orleans, LA

www.histoconvention.org/futuredates.cfm

Materials Science \& Technology (MS\&T)

September 29-October 3, 2019

Portland, OR

www.showsbee.com/fairs/42389-Materials-

Science-Technology-2019.html

Interdisciplinary Symposium on 3D

Microscopy

October 1-4, 2019

Engelberg, Switzerland

www.3dssom2019.ch

CIASEM

October 1-4, 2019

Buenos Aires, Argentina

http://ciasem2019.samic-argentina.org/registration

Neuroscience 2019

October 19-23, 2019

Chicago, IL

Sponsor: Society for Neuroscience

www.sfn.org

\section{AVS International}

Symposium \& Exhibition

October 20-25, 2019

Columbus, $\mathrm{OH}$

www.avs.org/symposium

\section{0}

Microscopy \& Microanalysis 2020

August 2-6, 2020

Milwaukee, WI

www.microscopy.org

\section{1}

Microscopy \& Microanalysis 2021

August 1-5, 2021

Pittsburgh, PA

www.microscopy.org

2022

Microscopy \& Microanalysis 2022

July 31-August 4, 2022

Portland, OR

www.microscopy.org

\section{3}

Microscopy \& Microanalysis 2023

July 24-28, 2023

Minneapolis, MN

www.microscopy.org

\section{4}

Microscopy \& Microanalysis 2024

July 28-August 1, 2024

Cleveland, $\mathrm{OH}$

www.microscopy.org

More Meetings and Courses

Check the complete calendar near the

back of this magazine.

\title{
The Importance of Spectrin: Can You Hear Me Now?
}

\author{
Stephen W. Carmichael \\ Mayo Clinic, Rochester, MN 55905 \\ carmichael.stephen@mayo.edu
}

Spectrin was first identified in red blood cells, where it functions as a scaffolding that is flexible and extensible, allowing the cells to deform in order to pass through tiny capillaries and then rebound to their original shape. It has since been determined that this cytoskeletal protein lines the intracellular surface of the plasma membrane of other eukaryotic cells including the hair cells of the inner ear. What were originally viewed as "hairs" are now known to be stereocilia. These specialized organelles are attached to the cuticular plate of what are still called hair cells. Nanoscale movements of these cilia in the inner ear are a critical step in converting physical movements into neural impulses that are interpreted by the brain as hearing and balance. The cochlea of the inner ear mediates hearing whereas the vestibular apparatus in the inner ear functions for balance.

The cuticular plate of hair cells in the cochlea is thought to be critical in hearing in mammals by securing stereocilia in place and providing them with the rigidity and support necessary for converting the mechanical energy of sound vibrations into electrical signals. The rootlets of stereocilia are electron-dense structures penetrating into the cuticular plate and forming an anchoring complex. This anchoring complex is a key structural component for stereocilia to withstand constant mechanical stresses and therefore plays a critical role in hearing. However, until now, the specific molecules that anchor the stereocilia and provide the necessary elasticity and flexibility have been elusive. In an elegant study published by Yan Liu, Jieyu Qi, Xin Chen, Mingliang Tang, Shuijin He, Renjie Chai, Guisheng Zhong and others, spectrin was identified as a critical element of the anchoring complex.

To determine the extent to which spectrin structures underpin the function of stereocilia, Liu et al. used super-resolution fluorescene imaging, which can provide molecular labeling specificity and live-cell imaging with nanoscale resolution. Experiments using immunostaining investigated the structure of both $\alpha$ II- and $\beta I I-s p e c t r i n$ in the cuticular plates of hair cells in mice. Using stimulated emission depletion imaging (one form of super-resolution microscopy) and transmission electron microscopy they obtained evidence that suggested spectrin forms cylinders that wrap around rootlets, functioning as a mechanical sensor during deflection of stereocilia. The intrinsic extensibility and elasticity of spectrin would provide this functional capability. These and other results suggested that $\alpha$ II- and $\beta I I-s p e c t r i n$ form flexible tetramers outlining the edges of the stereocilia rootlets of hair cells in the cuticular plate.

Since hair cells in the vestibule are not subject to the same mechanical stresses as those in the cochlea, Liu et al. compared the distribution of spectrin in the cuticular plate of hair cells in both the cochlea and the vestibule. Interestingly, spectrin was distributed in a meshwork arrangement in the vestibular hair cells, but no rings were observed.

Knockout mice were created that lacked $\beta I I-s p e c t r i n$ and, as expected, this molecule was completely deleted from the cuticular plate of these animals. $\alpha$ IIspectrin could be detected elsewhere in these animals but was nearly deleted from the cuticular plates, suggesting that $\beta \mathrm{II}$-spectrin may be required for the specific 


\section{Adding efficiency \\ to your fluorescence}

imaging.

ZEISS Celldiscoverer 7

\section{// INNOVATION}

MADE BY ZEISS

\section{Your automated platform for live cell imaging}

Imagine the ease of use and automation of a boxed microscope - combined with the image quality and flexibility of a classical research microscope. Imagine this system calibrates itself, detects and focuses your samples and adaptive optics adjust themselves automatically. ZEISS Celldiscoverer 7 is your reliable automated research platform. No matter if you work with 2D or 3D cell culture, tissue sections or small model organisms. With Celldiscoverer 7 you increase the efficiency of your research. You acquire better data in a shorter time. 

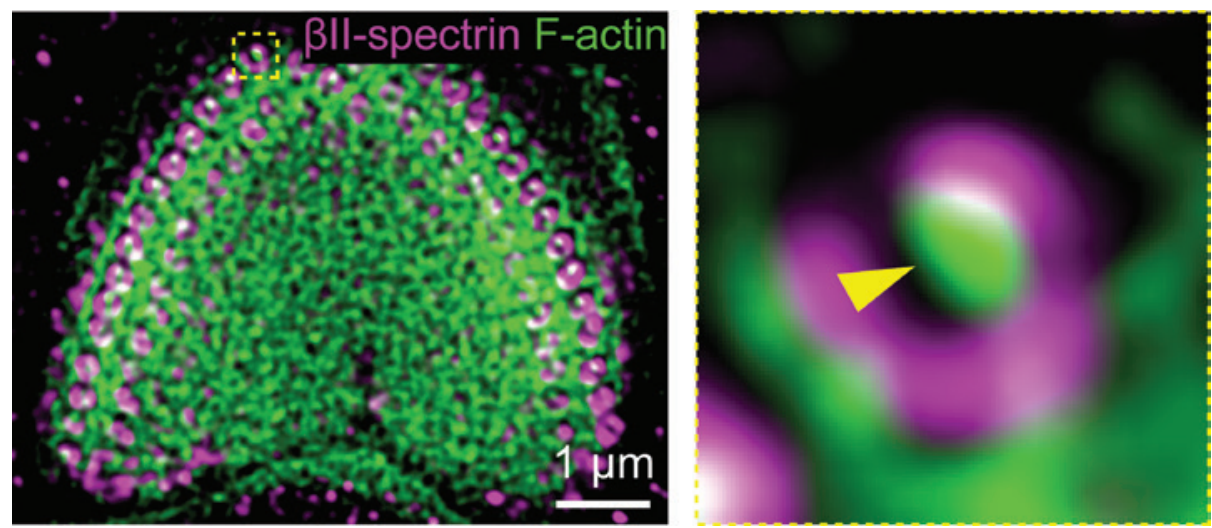

Figure 1: Representative two-color stimulated emission depletion images of $\beta I I-s p e c t r i n$ (magenta) and F-actin (cyan) in the cuticular plate of a hair cell. The yellow box at the top of the image on the left indicates the area of the image on the right.

location of $\alpha$ II-spectrin in the cuticlar plate of hair cells. Additional results suggested that $\beta \mathrm{II}$-spectrin is important for arranging three other proteins known to be normally associated with the base of stereocilia: F-actin (Figure 1), taperin, and FAM65B.

Hearing acuity can be determined in animals by the auditory brainstem response (ABR) where electrical signals are detected by electrodes placed near the ear. Mice displayed a severe hearing loss when $\beta I I$-spectrin was knocked out. Similarly in aging mice and mice that were subjected to a loud noise known to damage hearing, the ABR was diminished. Imaging studies demonstrated a marked decrease in both forms of spectrin in the cuticular plate of the hair cells. Also in these studies functional tests for balance found no significant loss of vestibular function. These results demonstrate a crucial role of spectrin in hair cell function for hearing, but not for balance. Further studies showed that spectrin also plays a critical role in the development of hearing in young mice. The study of Liu et al. used multiple approaches to provide a structural basis for understanding the function of spectrin in the auditory system.

\section{References}

[1] Y Liu et al., Sci Adv 5(4) (2019) 5:eaav7803.

[2] The author gratefully acknowledges Dr. Guisheng Zhong for reviewing this article.

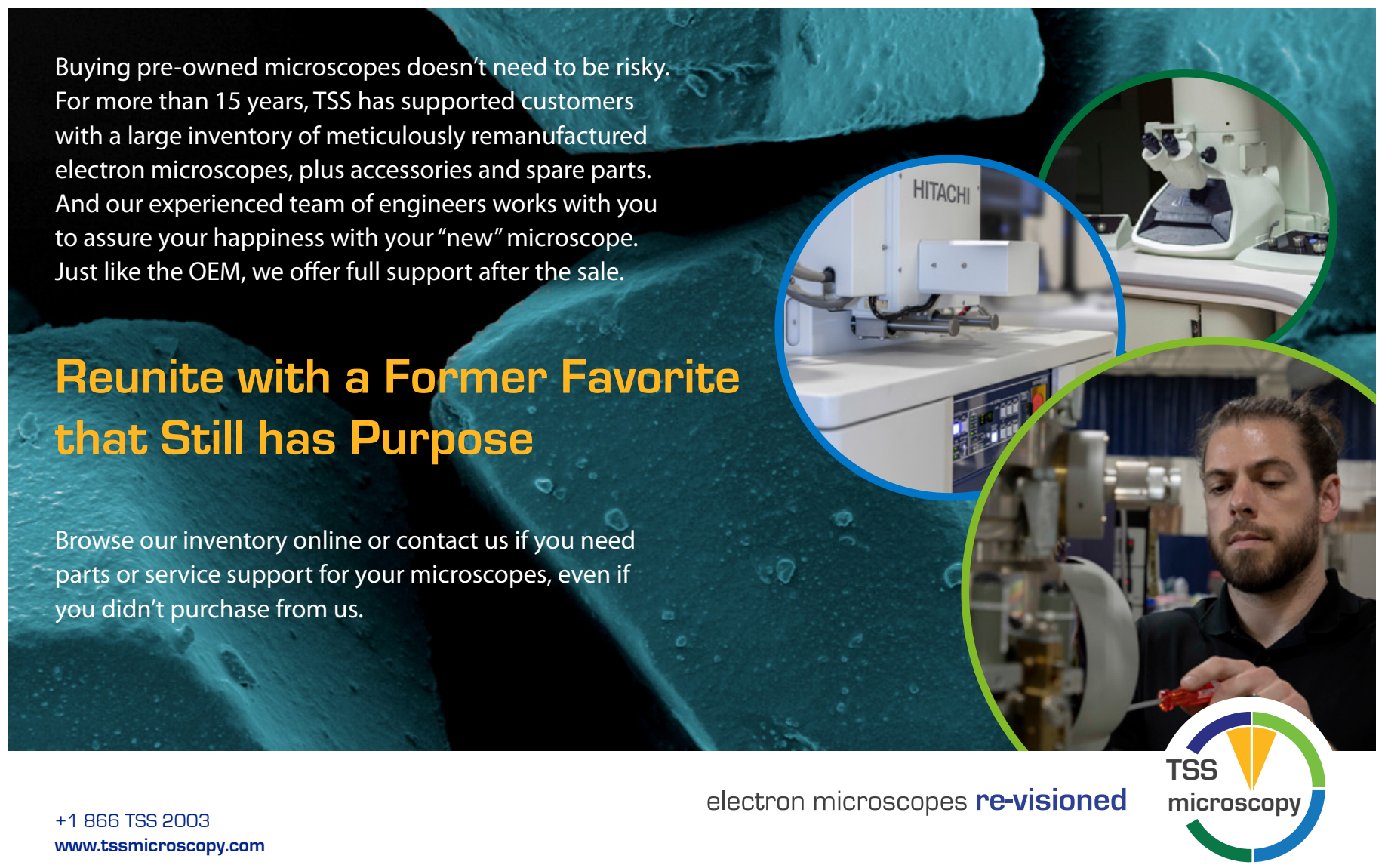


